\title{
Molecular Characterization and Antiviral Analyses of Porcine Type III Interferons
}

\author{
Yongming Sang, ${ }^{1}$ Raymond R.R. Rowland, ${ }^{2}$ and Frank Blecha ${ }^{1}$
}

Type III interferons (IFNs) are a family of recently identified antiviral cytokines. One to 3 paralogs have been identified in several species; however, little information is available about type III IFNs in pigs. We have identified 2 porcine type III IFNs, Sus scrofa IFN- $\lambda 1$ (SsIFN- $\lambda 1$ ) and SsIFN- $\lambda 3$, and determined their tissue expression profile and antiviral activities. Open reading frames of SsIFN- $\lambda 1$ and SsIFN- $\lambda 3$ are 576 and $588 \mathrm{bp}$, encoding 191 and 195 amino acid preproteins, respectively. In healthy pigs, $S s I F N-\lambda 3$ was primarily expressed in mesenteric lymph nodes and intestine, whereas expression of SsIFN- $\lambda 1$ was found in all tested tissues and was high in mesenteric lymph nodes, intestine, and liver. Porcine cells treated with the viral mimic, dsRNA, robustly increased SsIFN- $\lambda 3$ expression, with epithelial cells generally displaying the greatest response. Conversely, dsRNA-induced mRNA expressions of SsIFN- $\lambda 1$, SsIFN- $\alpha 1$, and SsIFN- $\beta$ were relatively weaker and delayed compared with SsIFN- $\lambda 3$. SsIFN- $\lambda 1$ and SsIFN- $\lambda 3$ peptides exerted similar but lower antiviral potency than SsIFN- $\alpha 1$ and SsIFN- $\beta$ against a porcine arterivirus and an adenovirus. These findings indicate that pigs have 2 type III IFN paralogs, which have antiviral activity and may serve as targets for modulation of the porcine hostpathogen interaction.

$I^{2}$ NTERFERONS (IFNS) ARE CLASSIFIED INTO 3 families, namely types I, II, and III (Ank and others 2006; Takeuchi and Akira 2009). A single type II IFN, ie, IFN- $\gamma$, is primarily produced by activated $\mathrm{T}$ and natural killer cells and is mainly associated with adaptive immunity (Ank and others 2006; Pestka 2007). Conversely, type I and type III IFNs consist of multiple members. For example, type I IFNs include several subtypes such as IFN- $\alpha$, IFN- $\beta$, IFN- $\varepsilon$, IFN- $\omega$, and IFN- $\kappa$ and are widely expressed in nucleated cells exerting early innate antiviral protection (Borden and others 2007; Pestka 2007). Type III IFNs are the most recently discovered IFN family and are encoded by 1-3 functional genes in various animal species. Designated IFN- $\lambda 1$, IFN- $\lambda 2$, and IFN- $\lambda 3$ in humans, they are also known as interleukin (IL)29, IL-28A, and IL-28B, respectively (Ank and Paludan 2009; Fox and others 2009).

Type III IFNs are distinct from type I IFNs in at least 4 features. First, they are encoded by genes with multiple exons (usually 5) in contrast to the single-exon genes for type I IFNs (Fox and others 2009). Second, the peptide structure of human IFN- $\lambda 3$ more closely resembles IL-22 of the IL-10 cytokine family rather than other IFNs (Gad and others 2009). Third, they use different receptors: IL-28RA/IL-10R2 rather than the IFNAR1/IFNAR2 receptors for type I IFNs (Kotenko and others 2003; Sheppard and others 2003). Fourth, unlike type I IFNs, which are widely expressed in nucleated cells, type III IFNs and their receptors are prominent in epithelial tissues, suggesting their involvement in epithelial antiviral immunity (Ank and others 2008; Sommereyns and others 2008; Wang and others 2009). Despite these distinctions, type III IFNs are induced through similar signal transduction pathways as type I IFNs (Ank and others 2006; Zhang and others 2008). For example, activation of signaling pathways mediated by Toll-like receptor (TLR)-3 and retinoic acid inducible gene I (RIG-I), which have been well characterized to induce IFN- $\alpha / \beta$ production, also induce type III IFNs in murine and human cells (Dumoutier and others 2004; Onoguchi and others 2007; Zhou and others 2009). Further, stimulated expression and antiviral potency of human type III IFNs have been associated with infections by several RNA and a few DNA viruses, including hepatitis $C$ virus, influenza $A$ virus, and cytomegalovirus (Ank and others 2006; Ank and Paludan 2009). Thus, type III IFNs comprise a group of newly identified antiviral cytokines that are functionally similar to type I IFNs and elicit first-line antiviral responses, especially in epithelial cells.

Annotation of available nucleic acid databases indicates that $1-3$ type III IFNs exist in most mammalian species (Fox and others 2009). Humans have 3 type III IFNs (IFN- $\lambda 1$, IFN-

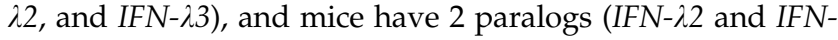
23) (Ank and Paludan 2009; Fox and others 2009). However, there is little information about type III IFNs in pigs. Because

Departments of ${ }^{1}$ Anatomy and Physiology and ${ }^{2}$ Diagnostic Medicine and Pathobiology, College of Veterinary Medicine, Kansas State University, Manhattan, Kansas. 
pigs are often used for comparative physiological and immunological studies and because porcine tissues and organs are often used for xenotransplantation, we sought to determine the type III IFN profile in pigs. Here we report the identification and initial characterization of 2 porcine
IFN- $\lambda s$-information that is fundamental for comparative investigations of type III IFNs in innate immunity.

Using bioinformatic annotation and experimental isolation as described previously (Sang and others 2005, 2008), 2 porcine IFN- $\lambda$ genes, similar to human IFN- $\lambda 1$ and IFN- $\lambda 3$,

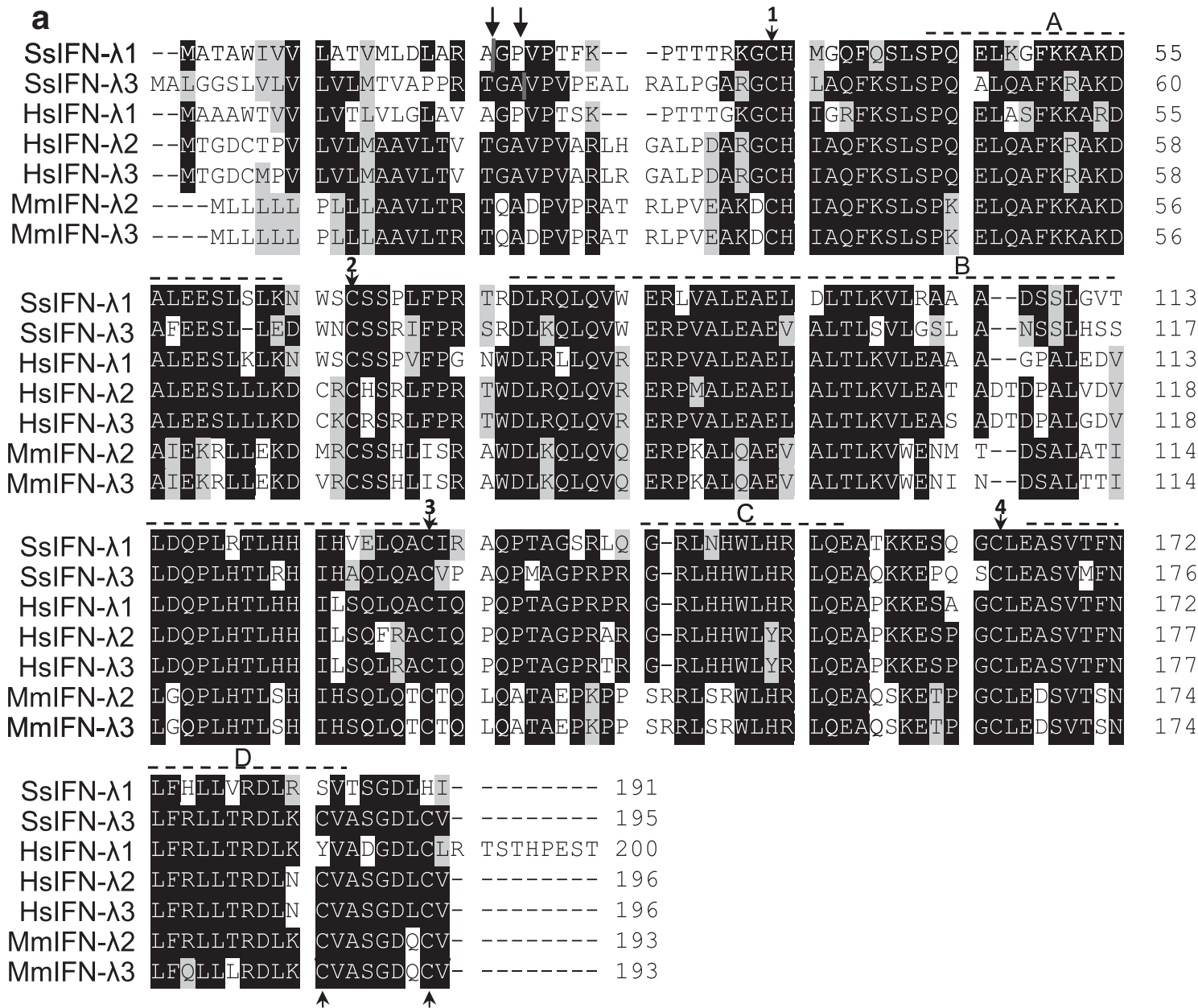

(5) $\quad$ (6)

b

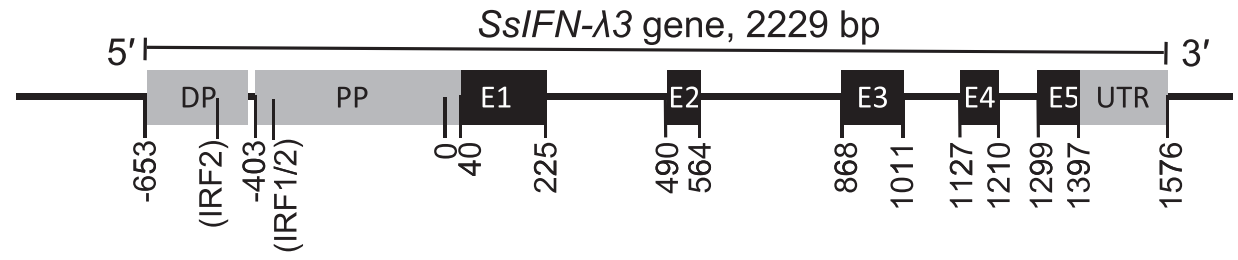

FIG. 1. Molecular identification of porcine type III interferons (IFNs). (a) Multiple sequence alignment of proproteins of porcine (Sus scrofa, Ss) IFN- $\lambda 1$ and IFN- $\lambda 3$ to human (Homo sapien, Hs) and murine (Mus musculus, Mm) type III IFNs. Residues with identities $>50 \%$ are shaded (light shading for similar residues and dark shading for identical residues). Predicted cleavage sites of signal peptidase on porcine type III IFNs are indicated by arrows at position 19 and 23 for SsIFN$\lambda 1$ and SsIFN- $\lambda 3$, respectively. The horizontal lines identify $4 \alpha$-helical regions (A to D) as predicted in SsIFN- $\lambda 3$. The numbered black arrows indicate conserved cysteine residues among all or some (numbers in parentheses) subtypes of aligned IFNs. Sequence alignment and protein predication were done with BioEdit (Hall 1999) and PredictProtein (Rost and others 2004). (b) Schematic diagram of SSIFN- $\lambda 3$ gene structure. Shown are predicted promoter regions (on minus strand) and a 5exon structure illustrated proportionally in scale (DP, distal promoter; PP, proximal promoter; E, exon; UTR, untranslated region). The predicted transcription start site (TSS) was defined as 0 position of the sequence and the positions of 2 punitive cis elements, potentially for IFN regulatory factors (IRF, position -383 and -491) are indicated in the predicted promoter region. Corresponding nucleotide positions are listed under the gene streamline. Gene sequence is available from GenBank (accession number of FJ853389). Gene structure was predicted with McPromoter (Ohler and Niemann 2001), GenomeScan (Yeh and others 2001), and radial basis functions-transcription start site (RBF-TSS) (Mahdi and Rouchka 2009). 
were identified and designated Sus scrofa IFN- $\lambda 1$ (SsIFN- $\lambda 1)$ (GenBank accession number FJ853390) and SsIFN- $\lambda 3$ (GenBank accession number GQ996936), respectively. The mRNA encoding preproteins of SsIFN- $\lambda 1$ and SsIFN- $\lambda 3$ have open reading frames of 576 and $588 \mathrm{bp}$ yielding preproteins of 191 and 195 amino acids and predicted N-terminal 19- and 23residue signal peptides, respectively (Fig. 1a). Similar to type I IFNs, mature SsIFN- $\lambda$ peptides are predicted to have $\alpha-$ helical structures consisting of 4 or 5 helices connected by short loops. Four and 6 conserved cysteine residues exist in SsIFN- $\lambda 1$ and SsIFN- $\lambda 3$ and are predicted to form 2 and 3 intramolecular disulfide bonds, respectively (Fig. 1a). One and $2 \mathrm{~N}$-glycosylation sites were also predicted in protein sequences of SsIFN- $\lambda 1$ (NWSC [one-letter amino acid notation] at positions 65-68) and SsIFN- $\lambda 3$ (NCSS at positions 7174 and NSSL at positions 111-114), respectively, indicating that N-glycosylation is likely associated with maturation of these IFN peptides. At the protein level, SsIFN- $\lambda 1$ and SsIFN$\lambda 3$ display $50 \%-70 \%$ identity to orthologs from other animal species. Our discovery of SsIFN- $\lambda 1$ was verified by an independent mRNA entry deposited in GenBank (accession number FJ455508). It is $99 \%$ identical to the SsIFN- $\lambda 1$ cDNA sequence we deposited with 1 nucleotide mismatch, perhaps reflecting gene polymorphism from different genotypes. Screening genomic and cDNA sources by in silico annotation or experimental isolation did not identify a porcine IFN- $\lambda 2$ ortholog. Thus, similar to mice, pigs likely have only 2 IFN$\lambda s$. In addition, we determined the gene structure of SsIFN$\lambda s$, as represented by $S s I F N-\lambda 3$ (Fig. 1b). The typical 5-exon gene structure (Fox and others 2009) was found in SsIFN- $\lambda 3$ with exon 1, 3, and 5 encoding most helical regions of the peptide (Fig. 1b). In the predicted promoter regions, there are several potential binding sites for IFN regulatory factors (Osterlund and others 2007) indicating potential involvement of these transcription factors in SSIFN- $\lambda$ expression (Fig. 1b) (Dumoutier and others 2004; Onoguchi and others 2007; Zhou and others 2009).

To determine how the development of porcine IFN- $\lambda \mathrm{s}$ relates to other animal type III IFNs, a phylogenic analysis was performed as described previously (Sang and others 2006) and it suggests that all animal type III IFNs likely arise from a common IFN- $\lambda$ ancestral gene. Most IFN- $\lambda 1$ orthologs clustered closely in a few leaves, whereas IFN- $\lambda 2$ and IFN- $\lambda 3$ orthologs were distributed in different leaves according to animal species or clades (Fig. 2). This suggests that IFN- $\lambda 1$ is phylogenically distant from other IFN- $\lambda s$, and as IFN- $\lambda 2$ and IFN- $\lambda 3$ diversified more recently, they are more similar. In addition to gene diversification within an animal species, coexistence of closely related IFN- $\lambda$ molecules across species implies a recent gene cross-species conversion (Fox and others 2009).

To examine the basal tissue expression profiles of porcine IFN- $\lambda s$, mRNA expression from 5 -week-old healthy pigs was examined using real-time reverse transcription (RT)polymerase chain reaction (PCR), as described previously (Sang and others 2005). Primers used are shown in Table 1. All animal procedures were approved by the Kansas State University Institutional Animal Care and Use Committee. Relative gene expression data in different tissues were normalized against critical threshold $\left(C_{t}\right)$ values of the housekeeping gene (glyceraldehyde 3-phosphate dehydrogenase) (Fig. 3) or calculated against control samples and presented as fold increase (Fig. 4). Expression of SsIFN- $\lambda 1$ was found in all tissues tested with the highest expression in mesenteric lymph nodes, duodenum, and liver. In contrast, SsIFN- $\lambda 3$ was clearly expressed in mesenteric lymph nodes and duodenum and only marginally detectable in other tissues (Fig.

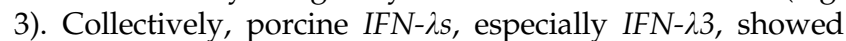
tissue-dependent expression; higher expression was found in the duodenum, liver, and mesenteric lymph nodes, and much lower expression in bone marrow, skin, spleen, and

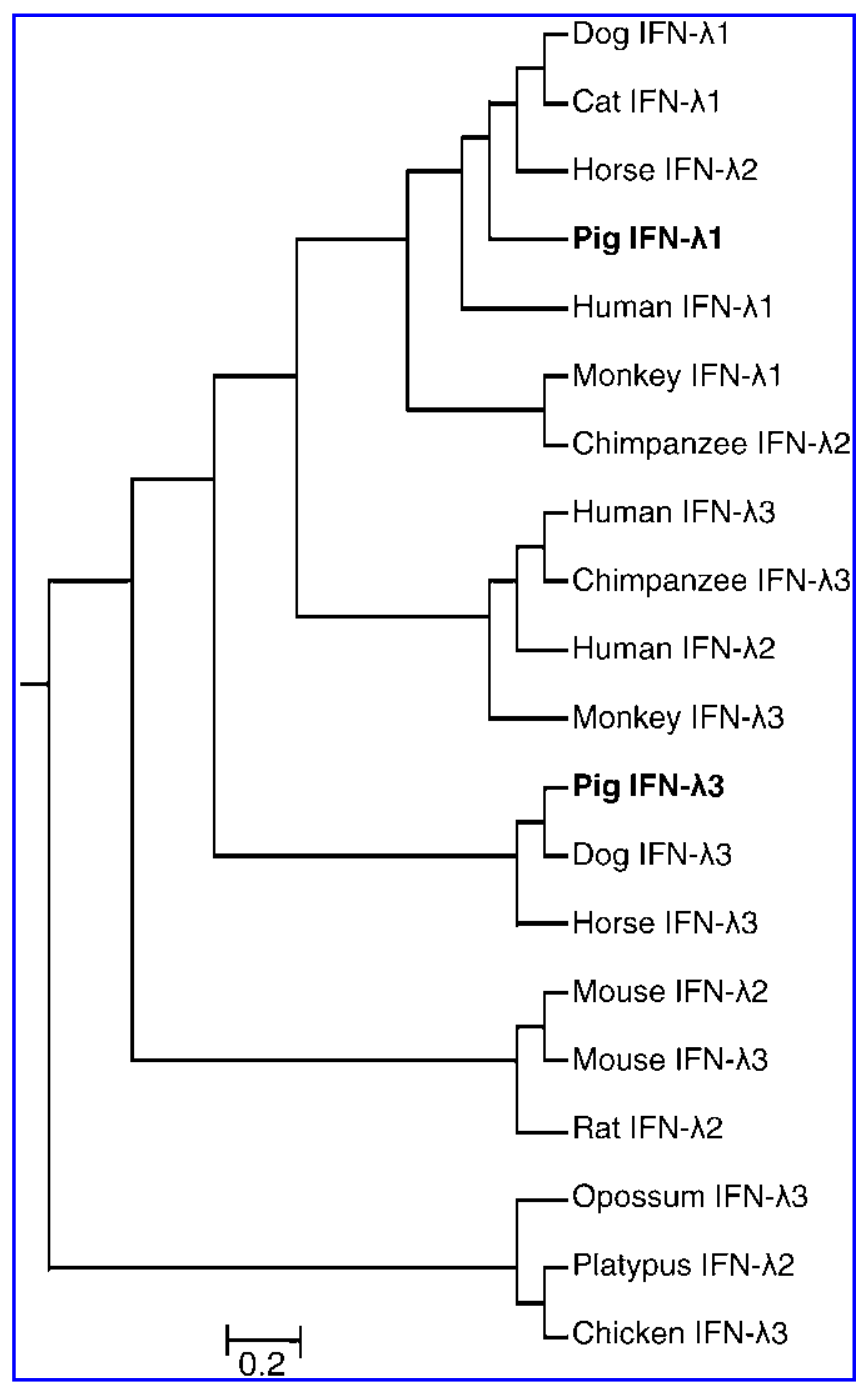

FIG. 2. Phylogenic relationship of porcine type III interferons (IFNs, bold text) and orthologs from other animal species. The neighbor-joining tree was constructed with CLUSTALW (Larkin and others 2007). GenBank accession numbers of aligned orthologs are as follows: dog (Canis familiaris) IFN- $\lambda 1$, NP_001108325 and IFN- $\lambda 3$, XP_855366; horse (Equus caballus) IFN- $\lambda 1$, XP_001501239 and IFN- $\lambda 3$, XP_001916418; cat (Felis catus) IFN- $\lambda 1, \quad N P \_001035770$; chicken (Gallus gallus) IFN- $\lambda 3$, NP_001121968; human (Homo sapiens) IFN- $\lambda 1$, AAI26184, IFN- $\lambda \overline{2}$, AAN86126 and IFN- $\lambda 3$, AAN86127; monkey (Macaca mulatta) IFN- $\lambda 1$, XP_001085768 and IFN- $\lambda 3$, XP_001086865; mouse (Mus musculus) IFN- $\lambda 2$, AAX58714 and IFN- $\lambda 3$, AAX58715; opossum (Monodelphis domestica) IFN- $\lambda 3$, XP_001368442; platypus (Ornithorhynchus anatinus) IFN- $\lambda 2, \quad X P \_001517931$; chimpanzee (Pan troglodytes) IFN- $\lambda 1$, XP_001135422 and IFN- $\lambda 3$, XP_001135462; Pig (Sus scrofa) IFN- $\lambda 1$, FJ853390 and SsIFN- $\lambda 3$, GQ996936; rat (Rattus norvegicus) IFN- $\lambda 2$, XP_001078329. 
Table 1. Cloning and Polymerase Chain Reaction Primers for Porcine Type III Interferons

\begin{tabular}{|c|c|c|c|}
\hline Subtype & & Primer name and sequence $\left(5^{\prime}-3^{\prime}\right)^{\mathrm{a}}$ & Product size $(b p)$ \\
\hline \multirow[t]{6}{*}{ SsIFN- $\lambda 1$} & Sense $^{\mathrm{b}}$ & gcсасcATGGCTACAGCTTGGATCGTGGT & \\
\hline & Antisense ${ }^{b}$ & TCAGATGTGCAAGTCTCCACTGGT & 582 \\
\hline & Antisense $^{b}$ & TCAagcgtaatctggaacatcgtatgggtaGATGTGCA & 609 \\
\hline & & AGTCTCCACTGGT & \\
\hline & Sense & ACATCCACGTCGAACTTCAGGCTT & \\
\hline & Antisense & TCAGATGTGCAAGTCTCCACTGGT & 209 \\
\hline \multirow[t]{5}{*}{ SsIFN- $\lambda 3$} & Sense $^{\mathrm{b}}$ & ACAGACATGGCCCTGGGT & \\
\hline & Antisense $\mathrm{b}^{\mathrm{b}}$ & TCAGACACACAGGTCTCCACT & 596 \\
\hline & Antisense $^{\mathrm{b}}$ & TCAagcgtaatctggaacatcgtatgggtaGACACAC & 623 \\
\hline & Sense & AAGAGGGCCAAGGATGCCTTTGAA & \\
\hline & Antisense & AGGCGGAAGAGGTTGAACATGACA & 374 \\
\hline
\end{tabular}

${ }^{a}$ Lowercase nucleic acids are added Kozak consensus sequences (Kozak 1987), or hemagglutinin-tag sequences (underlined) (Kolodziej and Young 1991)

${ }^{\mathrm{b}}$ Cloning primers.

SsIFN, Sus scrofa interferon.

testis. Because these tissues were from healthy animals without clinical symptoms of viral infection, it is possible that SsIFN- $\lambda$ s could be innate immune effectors stimulated during pathogen surveillance in the intestine, liver, and mesenteric lymph nodes. Because type III IFNs have been reported to primarily act on epithelial cells in vivo (Ank and others 2008; Sommereyns and others 2008; Wang and others 2009), future studies using antibodies to SsIFN- $\lambda$ s and their receptors may determine whether $\operatorname{SsIFN}-\lambda$ responses are highly restricted to the epithelial barriers of these porcine tissues.

In the next set of experiments, we investigated the regulatory expression of $S S I F N-\lambda$ s in 4 porcine cell lines stimulated with synthetic dsRNA (polyinosinic:polycytidylic acid),

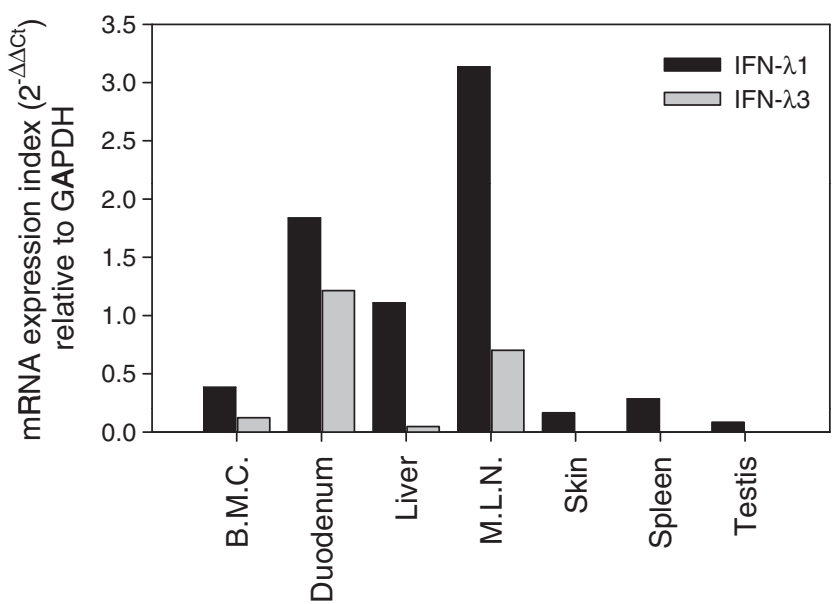

FIG. 3. Expression of Sus scrofa interferon- $\lambda 1$ (SsIFN- $\lambda 1)$ and $\operatorname{SsIFN}-\lambda 3$ in porcine tissues. Tissues were from 5 -weekold crossbred pigs without any clinical disease. Real-time RT-polymerase chain reaction (PCR) assays were conducted with gene-specific primers using total RNA (150 ng in $25 \mu \mathrm{L}$ PCR). Gene expression indexes $\left(2^{-\Delta \Delta C t}\right)$ were calculated by normalization against expression of a housekeeping gene (glyceraldehyde 3-phosphate dehydrogenase [GAPDH]) and average expression level of all tissues. B.M.C., bone marrow cells; M.L.N., mesenteric lymph nodes. Data represent 2 independent experiments with similar results. a well-tested molecular mimic of virus infection. Porcine cells at $60 \%-80 \%$ confluence ( 2 days after subculture) were treated with $10 \mu \mathrm{g} / \mathrm{mL}$ of dsRNA (Invivogen, San Diego, CA) for 3 and $24 \mathrm{~h}$. Cells were collected and total RNA was extracted using a Tri-Reagent procedure (Sigma-Aldrich, St. Louis, MO). Similar to our tissue expression findings, $S$ SIFN- $\lambda 1$ had higher basal expression in mock-treated control cells (data not shown). However, expression of $S s I F N-\lambda 3$ was robustly increased by dsRNA treatment in all 4 cell lines (Fig. 4). Among them, 2 epithelial cell lines, IPEC-J2 and PVD derived from porcine intestine and vas deferens, respectively (Sang and others 2005; Hagedorn and others 2007), showed the highest stimulation. Upregulation of SsIFN- $\lambda 1$ by dsRNA was only observed in the vas deferens epithelial cell line (PVD). The dsRNA-stimulated expression of SsIFN- $\lambda 3$ in all tested epithelial cells (PK-15, IPEC-J2, and PVD) was highest at $3 \mathrm{~h}$ after treatment and sharply attenuated at $24 \mathrm{~h}$ (Fig. 4). In contrast, stimulation in testis fibroblast cells (ST) was much lower compared with IPEC-J2 and PVD cells and did not decrease at $24 \mathrm{~h}$ (Fig. 4). For comparative purposes, we also evaluated dsRNA-stimulated expression of SsIFN- $\alpha 1$ and SSIFN- $\beta$. In the tested cell lines, basal expressions of $S s I F N-\alpha 1$ and SSIFN- $\beta$ in control samples were similar to $S s I F N-\lambda 1$, but $\sim 10-50$-fold greater than SsIFN- $\lambda 3$. However, stimulation with dsRNA significantly increased the expression of SsIFN- $\lambda$ s. Indeed, in most treated cells at both 3 and $24 \mathrm{~h}$ posttreatment, $S$ SIFN- $\lambda 3$ attained levels greater than SsIFN- $\alpha 1$ and SsIFN- $\beta$ (Fig. 4). Others have also observed that viral molecular mimics, including dsRNA, are stronger stimulators for human IFN- $\lambda$ expression (Ank and others 2006; Zhou and others 2009). Compared with the nonepithelial cells that were tested (ST), the 3 epithelial cell lines originating from kidney (PK-15), intestine (IPEC-J2), and vas deferens (PVD) all displayed dsRNA-stimulated peak expression of IFN- $\lambda 3$ at $3 \mathrm{~h}$ posttreatment, which was sharply attenuated at $24 \mathrm{~h}$. In addition, stimulated expression of IFN$\lambda 3$ was robustly increased in IPEC-J2 and PVD epithelial cells at $3 \mathrm{~h}$ post-dsRNA treatment (Fig. 3). Because synthetic dsRNA is a ligand for several innate immune receptors, including TLR-3, RIG-I, and melanoma differentiation-associated gene-5 (Takeuchi and Akira 2009), which mediate antiviral responses by inducing type I and type III IFNs, it 

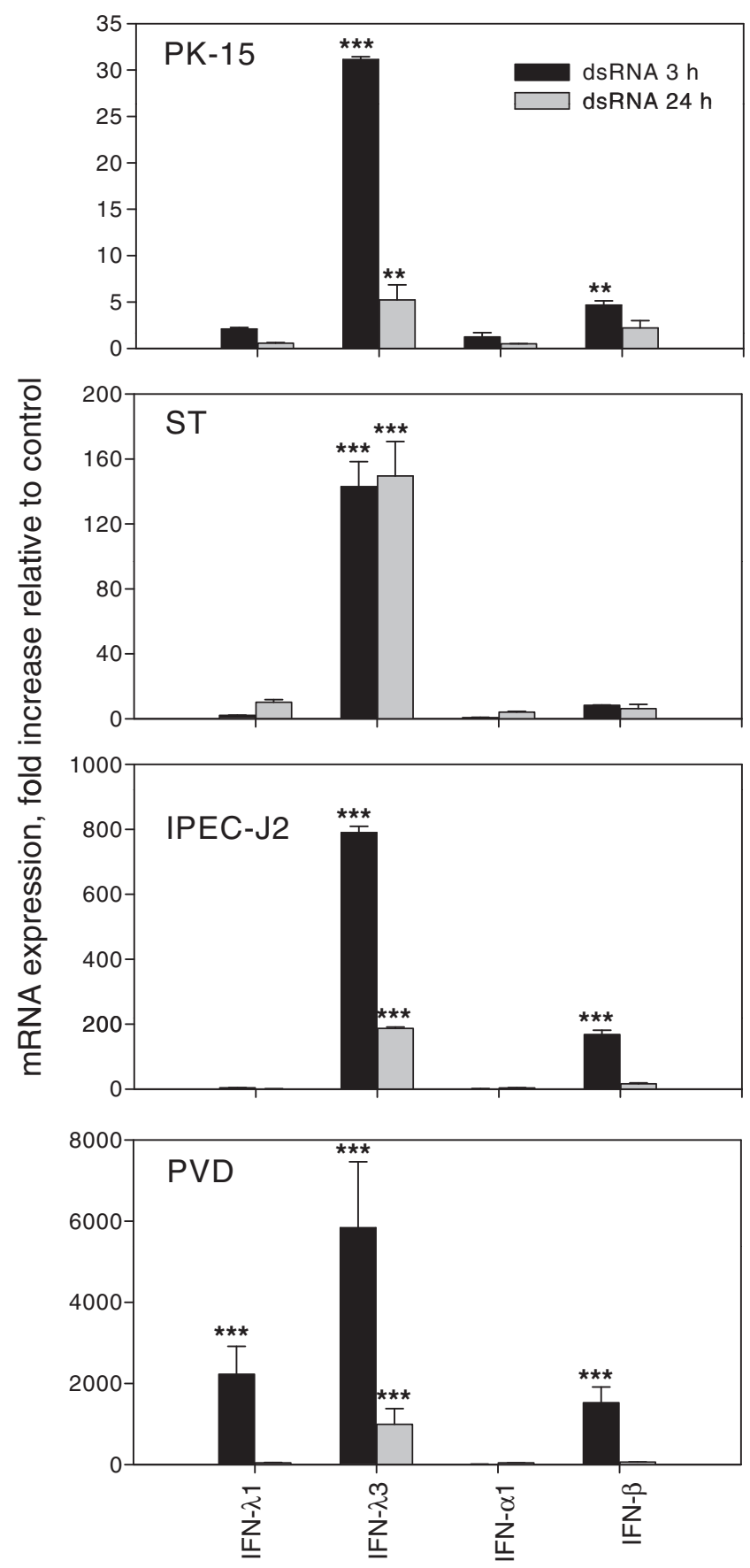

FIG. 4. The viral mimic, dsRNA, induces Sus scrofa interferon$\lambda s(S s I F N-\lambda s)$ expression in porcine kidney (PK-15), testis (ST), intestine (IPEC-J2), and vas deferens (PVD) cells. All cells are epithelial except ST, which are fibroblasts. Cells at $60 \%-80 \%$ confluence ( 2 days after subculture) were treated with dsRNA at $10 \mu \mathrm{g} / \mathrm{mL}$ for 3 or $24 \mathrm{~h}$. Total RNA was extracted from cells and 1-step real-time RT-polymerase chain reaction assays were conducted as in Fig. 3. Data are mean \pm standard error of mean; $n=3 ;{ }^{* *} P<0.01$ and ${ }^{* * *} P<0.001$.

will be informative to determine which receptor-mediated signaling pathway is most responsible for type III IFN expression in porcine epithelial cells, and if the distribution of receptors defines the production of type III IFN in different cell types.
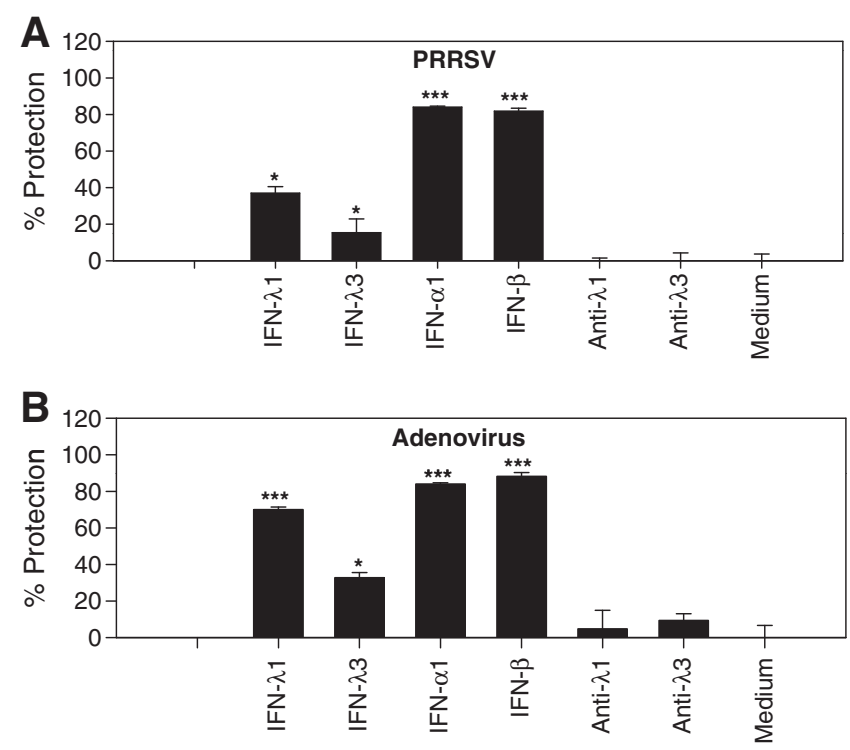

FIG. 5. Porcine interferon- $\lambda s$ (IFN- $\lambda s$ ) induce antiviral protection against porcine reproductive and respiratory syndrome virus (PRRSV) and adenovirus in porcine cells. Porcine alveolar macrophages (A) and PK-15 (B) cells were cultured in flat-bottomed 96-well plates and $10 \mu \mathrm{L}$ of serially diluted (1:10 to $1: 10^{6}$ dilutions of initial concentrated peptides at $10 \mu \mathrm{g} / \mathrm{mL}$ ) IFN- $\lambda$ peptides were added to wells with $90 \mu \mathrm{L}$ of predispensed fresh medium containing each virus. Shown here is antiviral protection of tested IFN peptides at a $10^{2}$ dilution $(0.1 \mu \mathrm{g} / \mathrm{mL})$. Negative and positive control wells included substitution of $10 \mu \mathrm{L}$ of IFN- $\lambda$ peptides with (1) Medium: medium collected from mock-transfected cells, (2) Anti- $\lambda 1$ or Anti- $\lambda 3$ : medium collected from cells transfected with Sus scrofa interferon $(S s I F N-\lambda)$ genes constructed in antisense orientation, and (3) IFN- $\alpha 1$ or IFN- $\beta$ : positive controls at $0.1 \mu \mathrm{g} / \mathrm{mL}$. Protective activity of IFNs on viral infections was examined using immunostaining for PRRSVAMs (24 h postinfection) (Sang and others 2008) and detection of green fluorescent protein-labeled adenovirus $(60 \mathrm{~h}$ postinfection) using fluorescent microscopy. Percentage of protection was calculated based on the ratio of virus-positive cells versus total cells. Approximately 2,000 cells were evaluated in each treatment. Data are mean \pm standard error of mean; $n=6,{ }^{*} P<0.05 ; n=3,{ }^{* * *} P<0.001$.

To evaluate the antiviral potency of SsIFN- $\lambda 1$ and SsIFN$\lambda 3$, we constructed and inserted their whole open reading frames (ORFs) into a mammalian cell expression vector (pcDNA $^{\text {TM} 3.3-T O P O ~}{ }^{\circledR}$; Invitrogen, Carlsbad, CA) as described previously (Sang and others 2008). Cloning primers are shown in Table 1 . The authenticity of the inserted cDNA was confirmed by sequencing, and the plasmids were transfected ( $\sim 70 \%$ transfection efficiency) into HEK293F cells cultured in serum-free medium. Constitutive expression of transfected SsIFN- $\lambda$ cDNA driven by the vector cytomegalovirus (CMV) promoter was estimated to be at $C_{t}$ values of $12-14$ using a real-time RT-PCR assay. SsIFN- $\lambda$ peptides were collected from the cell culture medium and concentrated 10-fold using 2 centrifugal filters (Centricon 50,000 and 10,000 NMWL; Millipore, Billerica, MA). To evaluate the IFN peptides, $20 \mu \mathrm{L}$ of concentrated peptides were resolved by gel electrophoresis (NuPAGE ${ }^{\circledR}$ Bis-Tris gel system; Invitrogen) and stained (Coomassie Blue G-250 solution; BioRad, Hercules, CA). Peptide concentrations were measured 
with a micro bicinchoninic acid protein assay (Pierce, Rockford, IL) and adjusted to $10 \mu \mathrm{g} / \mathrm{mL}$. Apparent molecular weights of SsIFN- $\lambda 1$ and SsIFN- $\lambda 3$ were $\sim 30$ and $35 \mathrm{kDa}$, respectively. Authenticity of IFN- $\lambda$ peptides was verified by incorporation of a hemagglutinin (HA) tag into their C-termini and staining using an immunoblotting procedure (Sang and others 2005) with an anti-HA monoclonal antibody (data not shown). The antiviral potency of overexpressed SsIFN- $\lambda$ peptides was examined in 2 porcine cells, primary alveolar macrophages and a kidney epithelial cell line (PK-15), against an arterivirus, porcine reproductive and respiratory syndrome virus (PRRSV), and an adenovirus, respectively. In brief, cells were cultured in flat-bottomed 96well plates and $10 \mu \mathrm{L}$ of IFN peptides serially diluted (1:10 to $1: 10^{6}$ dilutions of initial concentrated peptides at $10 \mu \mathrm{g} / \mathrm{mL}$ ) was added to each well with predispensed $90 \mu \mathrm{L}$ of fresh medium containing the indicated virus. Virus titers for infections were at a multiplicity of infection (MOI) of 0.1 tissue culture infections dose $\left(\mathrm{TCID}_{50}\right) / \mathrm{mL}$ for PRRSV and adenovirus. Negative and positive control wells included substitution of $10 \mu \mathrm{L}$ of IFN- $\lambda$ peptides with (1) minimum essential medium (MEM): medium collected from mocktransfected cells, (2) antisense constructs: medium collected from cells transfected with pcDNA3.3-antisense genes, and (3) IFN- $\alpha 1$ or IFN- $\beta$ : positive controls at $0.1 \mu \mathrm{g} / \mathrm{mL}$. The protective activity of IFNs against viral infections was determined using immunostaining (PRRSV, $24 \mathrm{~h}$ postinfection) and expression of fluorescent proteins (adenovirus, $60 \mathrm{~h}$ postinfection) (Sang and others 2008). SsIFN- $\lambda 1$ and SsIFN$\lambda 3\left(10^{2}\right.$ dilution; $\left.0.1 \mu \mathrm{g} / \mathrm{mL}\right)$ afforded $\sim 40 \%$ and $20 \%$ protection, respectively, against PRRSV infection in alveolar macrophages (Fig. 5A). The antiviral effect of both porcine IFN- $\lambda$ s was comparably weaker than the positive controls of SsIFN- $\alpha 1$ and SsIFN- $\beta$, which suppressed PRRSV infection in alveolar macrophages to $\sim 80 \%$. Negative controls that were used (cells treated with medium collected from nontransformed cells or from cells transformed with plasmids containing SsIFN- $\lambda 1$ and SsIFN- $\lambda 3$ in antisense orientation) showed slight or no suppression of PRRSV infection (Fig. $5 \mathrm{~A})$. The antiviral activity of SsIFN- $\lambda 1$ and SsIFN- $\lambda 3$ against adenovirus in PK-15 cells was $\sim 2$-fold higher than that against PRRSV in alveolar macrophages. Protection of PK-15 cells against adenovirus by SsIFN- $\alpha 1$ and SsIFN- $\beta$ was similar to protection observed in PRRSV-infected alveolar macrophages, ie, $\sim 80 \%-90 \%$ (Fig. 5B). However, at a lower concentration $\left(10^{3}\right.$ dilution), SsIFN- $\lambda 1$ and SsIFN- $\lambda 3$ only had marginal protection, whereas SsIFN- $\alpha 1$ and SsIFN- $\beta$ persisted with $80 \%-90 \%$ protection against adenovirus infection until $10^{5}$ dilution (data not shown). We also tested the anti-PRRSV potency of SsIFN- $\lambda 1$ and SsIFN- $\lambda 3$ in MARC-145 cells, a monkey model cell line for PRRSV replication and titration (Sang and others 2008). Using 10-fold serial dilution, we determined that SsIFN- $\lambda 1$ at $0.01 \mu \mathrm{g} / \mathrm{mL}$ provided nearly full protection against PRRSV, which was comparable to SsIFN- $\beta$. Conversely, a similar concentration of SsIFN- $\lambda 3$ only had partial $(\sim 20 \%)$ protective activity on MARC-145 cells (data not shown). Thus, although the 2 porcine type III IFNs had lower antiviral potency than porcine type I IFNs, they did induce antiviral protection in different porcine cells against PRRSV or adenovirus. This is similar to data with human type III IFNs that exerted similar but weaker antiviral activity than IFN- $\alpha$ and IFN- $\beta$ (Meager and others 2005).
In summary, we have identified the primary, if not all, members of the porcine type III IFN family. Porcine IFN- $\lambda s$ appear to display a tissue/cell type-dependent expression profile and exert similar but weaker antiviral activity than type I IFNs. The direct antiviral activity of porcine IFN- $\lambda s$ in porcine cells may indicate their potential for modulating antiviral immunity in pigs.

\section{Acknowledgments}

The authors thank Danielle Goodband for her technical expertise. This work was supported in part by grants from the USDA NRICGP (2006-35204-17337 and 2003-3520413704).

\section{Author Disclosure Statement}

No competing financial interests exist.

\section{References}

Ank N, Iversen MB, Bartholdy C, Staeheli P, Hartmann R, Jensen UB, Dagnaes-Hansen F, Thomsen AR, Chen Z, Haugen H, Klucher K, Paludan SR. 2008. An important role for type III interferon (IFN-lambda/IL-28) in TLR-induced antiviral activity. J Immunol 180:2474-2485.

Ank N, Paludan SR. 2009. Type III IFNs: new layers of complexity in innate antiviral immunity. Biofactors 35:82-87.

Ank N, West H, Paludan SR. 2006. IFN-lambda: novel antiviral cytokines. J Interferon Cytokine Res 26:373-379.

Borden EC, Sen GC, Uze G, Silverman RH, Ransohoff RM, Foster GR, Stark GR. 2007. Interferons at age 50: past, current and future impact on biomedicine. Nat Rev Drug Discov 6:975-990.

Dumoutier L, Tounsi A, Michiels T, Sommereyns C, Kotenko SV, Renauld JC. 2004. Role of the interleukin (IL)-28 receptor tyrosine residues for antiviral and antiproliferative activity of IL-29/interferon-lambda 1: similarities with type I interferon signaling. J Biol Chem 279:32269-32274.

Fox BA, Sheppard PO, O'Hara PJ. 2009. The role of genomic data in the discovery, annotation and evolutionary interpretation of the interferon-lambda family. PLoS ONE 4:e4933.

Gad HH, Dellgren C, Hamming OJ, Vends S, Paludan SR, Hartmann R. 2009. Interferon-lambda is functionally an interferon but structurally related to the interleukin-10 family. J Biol Chem 284:20869-20875.

Hagedorn TM, Carlin RW, Schultz BD. 2007. Oxytocin and vasopressin stimulate anion secretion by human and porcine vas deferens epithelia. Biol Reprod 77:416-424.

Hall TA. 1999. BioEdit: a user-friendly biological sequence alignment editor and analysis program for Windows 95/98/ NT. Nucleic Acids Symp Ser 41:95-98.

Kolodziej PA, Young RA. 1991. Epitope tagging and protein surveillance. Methods Enzymol 194:508-519.

Kotenko SV, Gallagher G, Baurin VV, Lewis-Antes A, Shen M, Shah NK, Langer JA, Sheikh F, Dickensheets H, Donnelly RP. 2003. IFN-lambdas mediate antiviral protection through a distinct class II cytokine receptor complex. Nat Immunol 4:69-77.

Kozak M. 1987. An analysis of $5^{\prime}$-noncoding sequences from 699 vertebrate messenger RNAs. Nucleic Acids Res 15:81258148.

Larkin MA, Blackshields G, Brown NP, Chenna R, McGettigan PA, McWilliam H, Valentin F, Wallace IM, Wilm A, Lopez R, Thompson JD, Gibson TJ, Higgins DG. 2007. ClustalW and ClustalX version 2. Bioinformatics 23:2947-2948. 
Mahdi RN, Rouchka EC. 2009. RBF-TSS: identification of transcription start site in human using radial basis functions network and oligonucleotide positional frequencies. PLoS ONE 4:e4878.

Meager A, Visvalingam K, Dilger P, Bryan D, Wadhwa M. 2005. Biological activity of interleukins-28 and -29: comparison with type I interferons. Cytokine 31:109-118.

Ohler U, Niemann H. 2001. Identification and analysis of eukaryotic promoters: recent computational approaches. Trends Genet 17:56-60.

Onoguchi K, Yoneyama M, Takemura A, Akira S, Taniguchi T, Namiki H, Fujita T. 2007. Viral infections activate types I and III interferon genes through a common mechanism. I Biol Chem 282:7576-7581.

Osterlund PI, Pietilä TE, Veckman V, Kotenko SV, Julkunen I. 2007. IFN regulatory factor family members differentially regulate the expression of type III IFN (IFN-lambda) genes. J Immunol 179:3434-3442.

Pestka S. 2007. The interferons: 50 years after their discovery, there is much more to learn. J Biol Chem 282:20047-20051.

Rost B, Yachdav G, Liu J. 2004. The PredictProtein server. Nucleic Acids Res 32(Web Server issue):W321-W326.

Sang Y, Patil AA, Zhang G, Ross CR, Blecha F. 2006. Bioinformatic and expression analysis of novel porcine $\beta$-defensins. Mamm Genome 17:332-339.

Sang Y, Ramanathan B, Ross CR, Blecha F. 2005. Gene silencing and overexpression of porcine peptidoglycan recognition protein long isoforms: involvement in beta-defensin-1 expression. Infect Immun 73:7133-7141.

Sang Y, Ross CR, Rowland RR, Blecha F. 2008. Toll-like receptor 3 activation decreases porcine arterivirus infection. Viral Immunol 21:303-313.

Sheppard P, Kindsvogel W, Xu W, Henderson K, Schlutsmeyer S, Whitmore TE, Kuestner R, Garrigues U, Birks C, Roraback J, Ostrander C, Dong D, Shin J, Presnell S, Fox B, Haldeman B, Cooper E, Taft D, Gilbert T, Grant FJ, Tackett M, Krivan W, McKnight G, Clegg C, Foster D, Klucher KM. 2003. IL-28, IL-29 and their class II cytokine receptor IL-28R. Nat Immunol 4:63-68.
Sommereyns C, Paul S, Staeheli P, Michiels T. 2008. IFN-lambda is expressed in a tissue-dependent fashion and primarily acts on epithelial cells in vivo. PLoS Pathog 4:e1000017.

Takeuchi O, Akira S. 2009. Innate immunity to virus infection. Immunol Rev 227:75-86.

Wang J, Oberley-Deegan R, Wang S, Nikrad M, Funk CJ, Hartshorn KL, Mason RJ. 2009. Differentiated human alveolar type II cells secrete antiviral IL-29 (IFN-lambda1) in response to influenza A infection. J Immunol 182:1296-1304.

Yeh RF, Lim LP, Burge CB. 2001. Computational inference of homologous gene structures in the human genome. Genome Res 11:803-816.

Zhang SY, Boisson-Dupuis S, Chapgier A, Yang K, Bustamante J, Puel A, Picard C, Abel L, Jouanguy E, Casanova JL. 2008. Inborn errors of interferon (IFN)-mediated immunity in humans: insights into the respective roles of IFN-alpha/beta, IFN-gamma, and IFN-lambda in host defense. Immunol Rev 226:29-40.

Zhou L, Wang X, Wang YJ, Zhou Y, Hu S, Ye L, Hou W, Li H, Ho WZ. 2009. Activation of toll-like receptor-3 induces interferon-lambda expression in human neuronal cells. $\underline{\text { Neu- }}$ roscience 159:629-637.

Address correspondence to: Frank Blecha, Ph.D.

Department of Anatomy and Physiology College of Veterinary Medicine

Kansas State University 228 Coles Hall

Manhattan, KS 66506

Phone: 785-532-4537

Fax: 785-532-4557

E-mail: blecha@vet.k-state.edu

Received 4 February 2010/Accepted 10 April 2010 

This article has been cited by:

1. Yongming Sang, Raymond R. R. Rowland, Frank Blecha. 2011. Interaction between innate immunity and porcine reproductive and respiratory syndrome virus. Animal Health Research Reviews 12:02, 149-167. [CrossRef]

2. Fayna Díaz-San Segundo, Marcelo Weiss, Eva Perez-Martín, Marla J. Koster, James Zhu, Marvin J. Grubman, Teresa de los Santos. 2011. Antiviral activity of bovine type III interferon against foot-and-mouth disease virus. Virology 413:2, 283-292. [CrossRef] 